\title{
Uso de la ecografía para el diagnóstico y seguimiento de la gestación en la cabra Murciano-Granadina
}

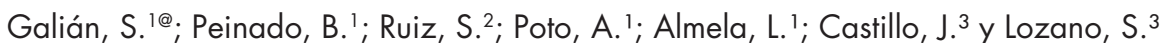

\author{
'Instituto Murciano de Investigación y Desarrollo Agrario y Alimentario (IMIDA). Murcia. España. \\ ${ }^{2}$ Fisiología de la Reproducción. Universidad de Murcia. España. \\ ${ }^{3}$ Asociación Española de Criadores de la Cabra Murciano - Granadina. Jumilla. España.
}

\section{PalABRAs ClAVE}

Caprino.

Doppler.

Ecografía.

Gestación.

Tres dimensiones.

\section{RESUMEN}

Se realiza una sucinta revisión en un intento de profundizar y mejorar la precocidad del diagnóstico ecográfico de la gestación. La descripción de los principios en los que se basa la aplicación de los ultrasonidos sobre las partes anatómicas del aparato genital de las hembras caprinas con indicación de las nuevas metodologías que en la actualidad pueden aplicarse, como es el caso de la apreciación de la circulación sanguínea en el desarrollo e implantación del embrión y del feto. Para ello se muestran en distintas coloraciones desde la formación de vesículas germinativas hasta el latido cardiaco en los estadios precoces de la gestación. La aplicación del sistema Doppler y el efecto de tres dimensiones en las imágenes ecográficas pueden ser de gran ayuda, no solo en el diagnóstico de la gestación sino también en el desarrollo embrionario. Se exponen imágenes sobre las gestaciones obtenidas en cabras Murciano Granadinas en distintos días tras la inseminación donde se percibe desde la aparición de líquidos indicadores de gestación hasta a presencia de órganos en distintos estadios de desarrollo.

\section{Ultrasound of gestation in Murciano Granadina goats}

\section{SUMMARY}

\section{ADDITIONAL KEYWORDS}

Doppler.

Gestation.

Goat.

Three dimensions.

Ultrasound.

INFORMATION

Cronología del artículo.

Recibido/Received: 18.05.2020

Aceptado/Accepted: 23.07.2020

On-line: 15.01.2021

Correspondencia a los autores/Contact e-mail:

sgalianarnaldos@gmail.com
A brief review is made in an attempt to deepen and improve the precocity of the ultrasound diagnosis of pregnancy. The description of the principles on which the application of ultrasound is based on the parts of the female genital tract of goats, with an indication of the new methodologies that can currently be applied, as is the case with the appreciation of blood circulation in the development and implantation of the embryo and the foetus. For this they are shown in different colorations from the formation of germinative vesicles to the heartbeat in the early stages of gestation. The application of the Doppler system and the effect of three dimensions on ultrasound images can be of great help, not only in the diagnosis of gestation but also in embryonic development. Images are exhibited on the pregnancies obtained in Murciano Granadina goats in different days after insemination where it is perceived from the appearance of gestation signs to the presence of organs at different stages of development.

\section{INTRODUCCIÓN}

La ultrasonografía es una técnica de exploración de los órganos internos del cuerpo mediante la emisión de ondas de sonido de alta frecuencia (ultrasonidos). Son aquellas ondas de sonido cuya frecuencia es superior a la audible por el oído humano, es decir, por encima de los $20.000 \mathrm{~Hz}$ (Hertzios). Aplicada al campo de la reproducción, la ultrasonografía es útil para la revisión del tracto reproductivo y visualización de anomalías, detección de gestación en los primeros estadios, esti- mación de la edad gestacional y fecha de parición, el número de embriones, determinación del sexo fetal, estados de pseudogestación o muerte fetal. El conocimiento, de todos estos parámetros, permitirá al ganadero mejorar el manejo reproductivo de sus animales, ya que le proporcionará conocimiento si las hembras presentan patologías en su sistema reproductor, cuántas hembras están gestantes, conocer el número de gestaciones múltiples para adecuar la dieta y suplementos alimenticios en caso de ser necesario, así como estimar la fecha de parto y el número de individuos de 
cada sexo esperado. También será útil para detectar y actuar en los casos en los que haya pseudogestación o se visualice muerte fetal, con el tratamiento que sea necesario aplicar a cada hembra.

El objetivo de este trabajo ha sido repasar la técnica de la ultrasonografía aplicada a la reproducción, concretamente en la especie caprina, para determinar cuándo ésta nos va a permitir el conocimiento de un diagnóstico fiable de gestación, así como número de fetos y viabilidad de los mismos. La aplicación de la información que nos proporcione permitirá un mejor funcionamiento de la explotación ganadera.

\section{PRINCIPIOS DE LA ULTRASONOGRAFÍA}

El funcionamiento consiste en una corriente eléctrica que llega al transductor (sonda) produciendo una vibración en sus cristales, denominados cristales piezoeléctricos. Éstos convierten los pulsos eléctricos en ondas sonoras que viajan a través de los órganos en estudio. Los tejidos tienen la capacidad, según su densidad y organización, de reflejar estas ondas de sonido, y el eco resultante es recibido por el transductor y convertido nuevamente en corriente eléctrica. Dentro del equipo esta corriente es decodificada y transformada en imágenes bidimensionales en tonos de grises del blanco al negro (Bidinost et al., 1999; Sánchez y Alfonso, 2000). Es una técnica segura, rápida y repetible sin causar daño al animal.

Como hemos comentado es la emisión y recepción de ondas sonoras la que, mediante transformación en electricidad nos va a proporcionar una imagen. Las ondas sonoras penetran en los tejidos y son devueltas como ecos, que dependen de la densidad y organización del mismo. Los líquidos no reflejan, o reflejan poco, las ondas ultrasónicas, por lo que proporcionan imágenes de color negro; conocidos como anecogénicos (Ramírez-Videla et al. 2009).

Tejidos densos reflejan mayor cantidad de ondas, dando imágenes en una escala de grises hasta llegar al color blanco que proporciona el tejido óseo, denominados hipoecogénicos o hiperecogénicos, según el grado de color blanco que proporcionan (Griffin y Ginter, 1992; Fricke, 2002).

Existen diferentes modos de ultrasonografía. El modo A, no se utiliza en reproducción y se basa en la emisión y recepción por el mismo transductor de un único haz de ultrasonidos. Los ecos devueltos son representados en forma de picos sobre una línea basal, en función de su amplitud y su distancia al receptor. Se obtiene un mapeo unidimensional en forma de gráfica, similar a la de un osciloscopio, de las interfases entre tejidos a lo largo de la línea de propagación del rayo de ultrasonidos (Giraldo, 2003).

El modo M, a lo largo de la línea que representa el haz ultrasónico se observarán los ecos como puntos de brillo de distinta intensidad, siendo la distancia entre ellos también proporcional al tiempo que tardan en ser recibidos. Esta línea de puntos es presentada en el monitor de forma continua a lo largo del tiempo, lo que significa que se pueden seguir con precisión los movimientos a lo largo del tiempo, por lo que es muy utilizado en ecocardiografía (Díez, 1992).
Actualmente en medicina veterinaria, los ecógrafos más utilizados son los llamados de modo B y tiempo completo (o real). Se refieren a que la imagen obtenida es un corte bidimensional del órgano en estudio formada por pequeños puntos de diferente densidad y que los impulsos se van transmitiendo constantemente y así se obtiene una visión instantánea de los tejidos examinados (Groenenberg et al., 2016). El poder de resolución del equipo, esto es, la capacidad de diferenciar entre dos puntos próximos entre sí, depende de la frecuencia de las ondas de sonido. Las más utilizadas son 3.5, 5.0 y 7.5 MHz (Megahertzios, $1 \mathrm{MHz}=1.000 .000$ de ondas de sonido/segundo). Frecuencias altas (7.5 $\mathrm{MHz}$ ) proporcionan imagen con mejor detalle (mayor resolución), pero tienen poco poder de penetración. Se usan principalmente en pequeños animales (Sánchez y Alfonso, 2000) pero también son utilizados, junto con los de $5.0 \mathrm{MHz}$ para el examen de ovarios, útero y preñeces tempranas. Para gestaciones avanzadas se recomienda usar transductores de $3.5 \mathrm{MHz}$ (Bo y Caccia, 2000). Los transductores o sondas se clasifican según la disposición de sus cristales piezoeléctricos. En los transductores lineales, los cristales se disponen en línea y emiten haces paralelos de ultrasonidos, por lo que se obtiene una imagen rectangular. Ofrecen una imagen amplia del campo cercano, pero la desventaja de necesitar un gran área de contacto (Díez, 1992). En reproducción son utilizados para examen por vía rectal. Una variante del transductor lineal es el convexo o cónvex, que también presenta los cristales en línea, pero curvada (Moreno et al., 1999). La superficie de contacto es convexa, lo que facilita el acoplamiento sobre la superficie del animal. Se utiliza para examen transabdominal (Moreno et al.1999). Los transductores sectoriales emiten haces divergentes de ultrasonidos, dando una imagen en abanico. Su principal ventaja es que la superficie de contacto es mínima, pero tiene como desventaja que el campo de visión es muy reducido (Díez, 1992). Su uso en reproducción es para análisis endovaginal.

\section{USO DE LA TECNOLOGÍA DOPPLER}

En la ultrasonografía Doppler, la imagen obtenida es el resultado de las ondas sonoras siendo reflejadas por estructuras en movimiento, como por ejemplo los eritrocitos moviéndose en el interior de vasos sanguíneos (Brant y Dougherty, 2012; Pellerito y Polak, 2012). Cuando los eritrocitos se mueven hacia el transductor, la frecuencia de retorno de las ondas de ultrasonido es mayor que la de ondas emitidas. La imagen se traduce como fluido sanguíneo de color rojo. Cuando los eritrocitos se alejan del transductor, la frecuencia de las ondas reflejadas es más pequeña que la de las ondas emitidas. El flujo sanguíneo es visualizado entonces de color azul (Petridis et al., 2017). De esta manera la ecografía Doppler permite una evaluación inmediata de la perfusión vascular de un tejido u órgano. Es decir, nos permite ver la irrigación de un determinado órgano de interés, visualizando el flujo sanguíneo en color rojo y azul, según su dirección de movimiento (Ginther, 2007). Aplicado a la reproducción resulta muy útil para determinar viabilidad fetal, ya que nos permite visualizar el latido cardiaco del embrión, además del flujo de cordón umbilical entre el embrión y la placenta 
(Serin et al. 2010; Endorgan, 2012). Hay autores que han utilizado la tecnología Doppler para determinar si hay o no gestación en estadios muy tempranos (días 17-21) mediante la evaluación de la vascularización del cuerpo lúteo (CL) (Arashiro et al., 2018; Cosentino et al., 2018). Así, CL en los que no se observa vascularización entre los días 15-17, puede afirmarse que el proceso de luteólisis ha comenzado y clasificar a esa hembra como no preñada.

\section{ECOGRAFÍA PARA DETECTAR GESTACIÓN EN LA ESPECIE CAPRINA}

Tradicionalmente la gestación en caprinos era detectada por el retorno a celo de las hembras no gestantes (Goel \& Agrawal, 1992), o bien por palpación manual en los flancos de la hembra para detectar la presencia del feto, obligándole a moverse dentro del líquido amniótico (Taverne \& Noakes, 2009). Estos métodos tienen el inconveniente de que durante la época de anestro estacionario es difícil que las hembras retornen al estro, o esperar a la segunda mitad de la gestación para poder detectar movimientos fetales por palpación (Memon \& Ott, 1980; Taverne \& Noakes, 2009)

El uso de la ecografía ha revolucionado el diagnóstico de la gestación, ya que permite detecciones tempranas de esta. Algunos autores reportan que incluso en día 16-18 de la gestación ya es posible visualizar una estructura anecogénica en el tejido uterino que correspondería con la vesícula embrionaria, primer signo ecográfico de gestación, aunque con estos días no es posible visualizarla en el 100\% de los casos (Santiago- Moreno et al. 1995a,b; Martínez et al. 1998). La mayoría de autores detectan en la especie caprina vesícula embrionaria aproximadamente en el día 25-26 de la gestación prácticamente en el 100\% de los casos, realizando ecografía transrectal o transvaginal (Basaran 1999; Gonzales et al. 2004). En este mismo día podemos ver el embrión y el latido cardiaco puede ser detectado mediante el sistema Doppler. El recuento embrionario puede hacerse también en esta fecha (Karen et al., 2004) aunque diversos autores consideran que es más certero realizarlo a partir del día 40 (Lavoir y Taverne, 1989; Medan y abd El-Aty, 2010), para evitar la subestimación del número de embriones que aún no son visualizados claramente.

\section{ECOGRAFÍA EN EL DIAGNÓSTICO DE LA GESTACIÓN DE LA CABRA MURCIANO-GRANADINA. RESULTA- DOS PRELIMINARES.}

En el Instituto Murciano de Investigación y Desarrollo Agrario y Alimentario se está realizando un estudio de toda la gestación caprina de la raza MurcianoGranadina. Este estudio se comenzó antes incluso de la propia gestación, mediante la evaluación del aparato genital de hembras de la raza, para visualizar mediante ecografía vaginal el estado de los ovarios, con sus respectivos folículos y cuerpos lúteos, como del útero. El equipo utilizado fue Voluson 730 expert, con sonda endovaginal de 5.0 MHz. Las ecografías se realizaron con el animal en decúbito supino en una camilla, sin necesidad de sedación. Pudimos visualizar los ovarios como estructuras redondeadas, laterales a la vejiga urinaria, con la presencia en ellos de estructuras circu- lares anecogénicas de diferente diámetro, los folículos, según el momento del ciclo (Figuras 1 y 2). En algunos casos pudimos detectar la presencia de CL como una masa circular dentro del ovario, ligeramente menos ecogénica que el tejido de alrededor (Figura 3). Craneal a la vejiga se localiza el útero, observándose como estructuras circulares de diferente textura al tejido que lo

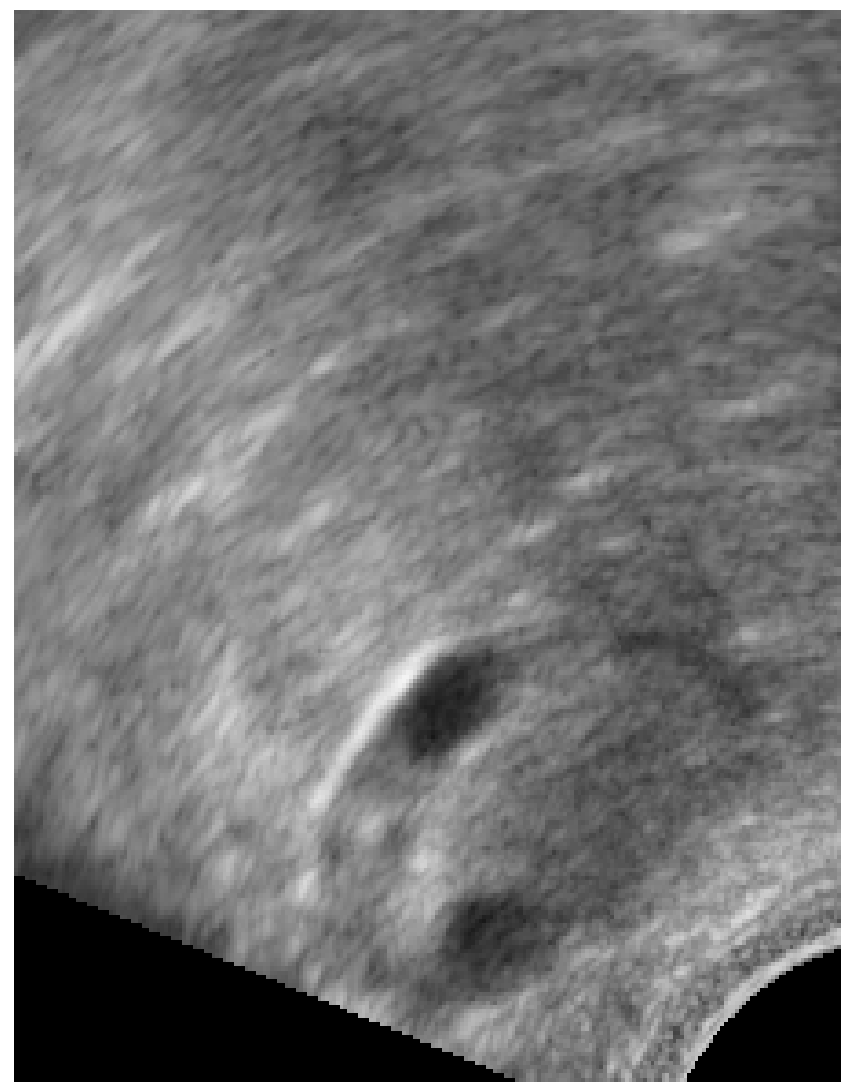

Figura 1. Ovario de hembra caprina no gestante. Estructuras redondeadas anecogénicas (negras) corresponden a los folículos (Ovary of non-pregnant caprine female. Anechogenic (black) rounded structures correspond to the follicles.)

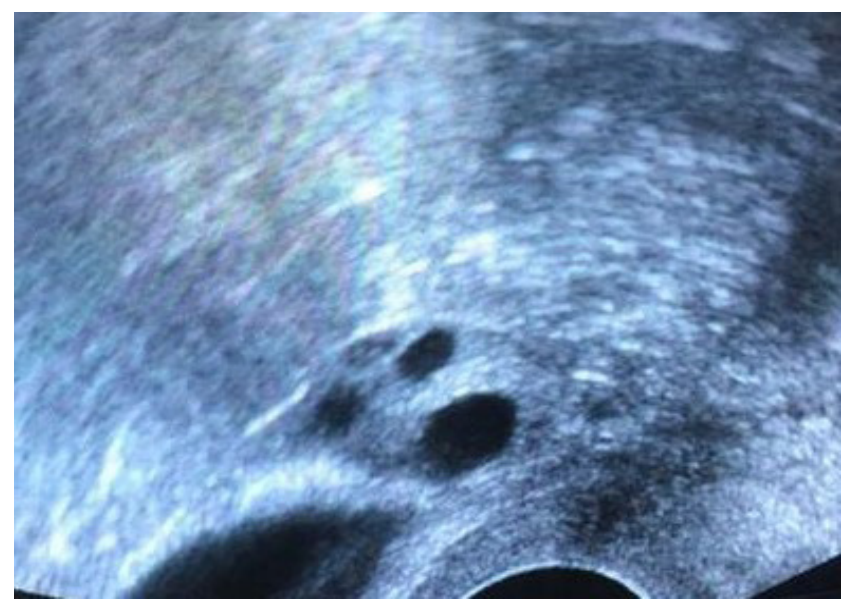

Figura 2. Ovario de hembra caprina no gestante. Estructuras redondeadas anecogénicas (negras) corresponden a los folículos (Ovary of non-pregnant caprine female. Anechogenic (black) rounded structures correspond to the follicles.) 


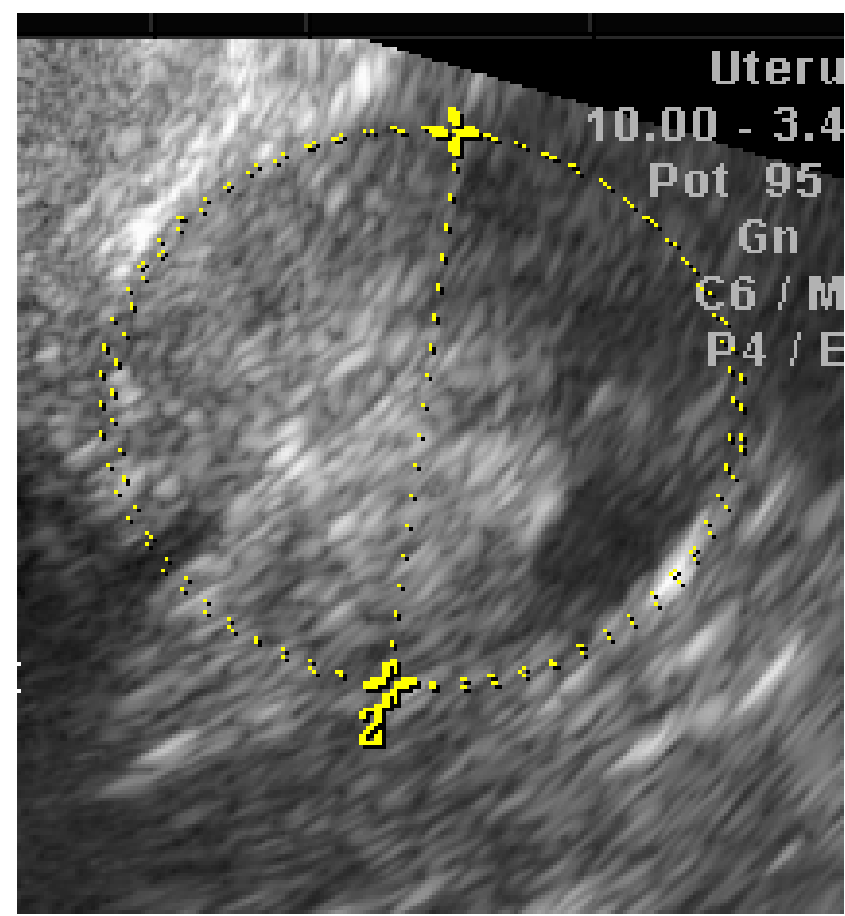

Figura 3. Ovario (interior círculo amarillo) con folículos, las flechas señalan un cuerpo lúteo (Ovary (inner yellow circle) with follicles, arrows point to a luteal body.)

rodea, que se corresponden con cortes transversales de los cuernos uterinos (Figuras 4 y 5).

Se seleccionaron 11 cabras multíparas que no presentaban patologías en su aparato reproductor. Las hembras fueron inseminadas artificialmente con semen de machos de raza Murciano Granadina, tras estimulación hormonal con esponjas intravaginales a base de progestágenos e inyección de prostaglandina f2 alfa (PF2 $\alpha$ ) y gonadotropina coriónica equina (eCG). (Poto et al., 1995; Peinado y Sánchez, 1998) El estudio ecográfico comenzó 14 días después de la inseminación, realizándose cada dos días hasta el día 30, posteriormente fueron hechas cada 5 días.

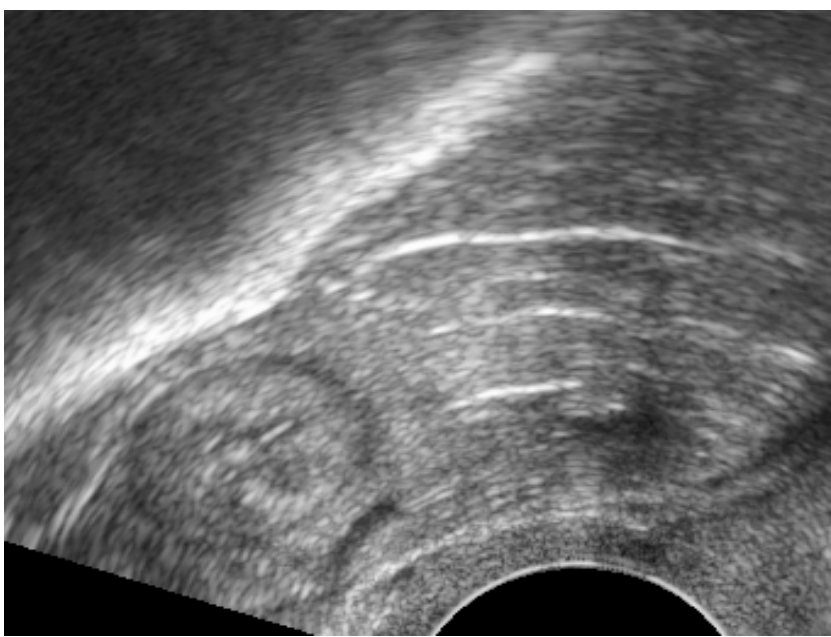

Figura 4. Corte transversal cuerno uterino no gestante (flecha) (Cross-section non-pregnant uterine horn (arrow)).

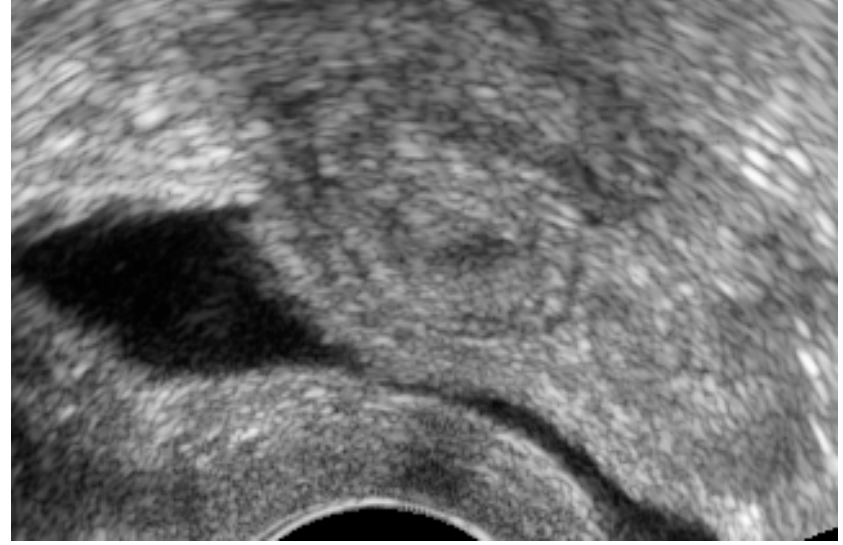

Figura 5. Corte transversal cuerno uterino no gestante (flecha). La estrella azul señala la vejiga urinaria (Cross-section non-pregnant uterine horn (arrow). The blue star points to the urinary bladder.)

En día 18, pudimos visualizar en algunas hembras la primera imagen anecogénica que podría corresponder a la vesícula embrionaria. No obstante, todavía la imagen era confusa y generaba dudas para dar un diagnóstico certero de gestación. Además, no era posible localizarla en todas las hembras que resultaron gestantes. En día 23, la vesícula embrionaria se detectó en las hembras que resultaron gestantes, aunque el diagnóstico era menos claro en algunas de las que resultaron vacías, pudiendo incluirse como preñadas hembras que resultaron no estarlo. En día 25 ya no hubo error, la vesícula fue localizada en el $100 \%$ de los casos, intuyéndose el embrión en su interior. El día 30 se detectó un crecimiento embrionario muy notable. Se pudo medir el embrión, (aproximadamente 1,75 cm de longitud cráneo-caudal, LCC) y ya se detectó latido cardiaco mediante sistema Doppler. En día 35 se estimó el número de embriones, aunque en algunos casos fue modificado en día 40, (Figuras 6-13) siempre por subestimación del número de embriones contados. En estas fechas el embrión mide sobre los $2 \mathrm{~cm}$ de LCC y

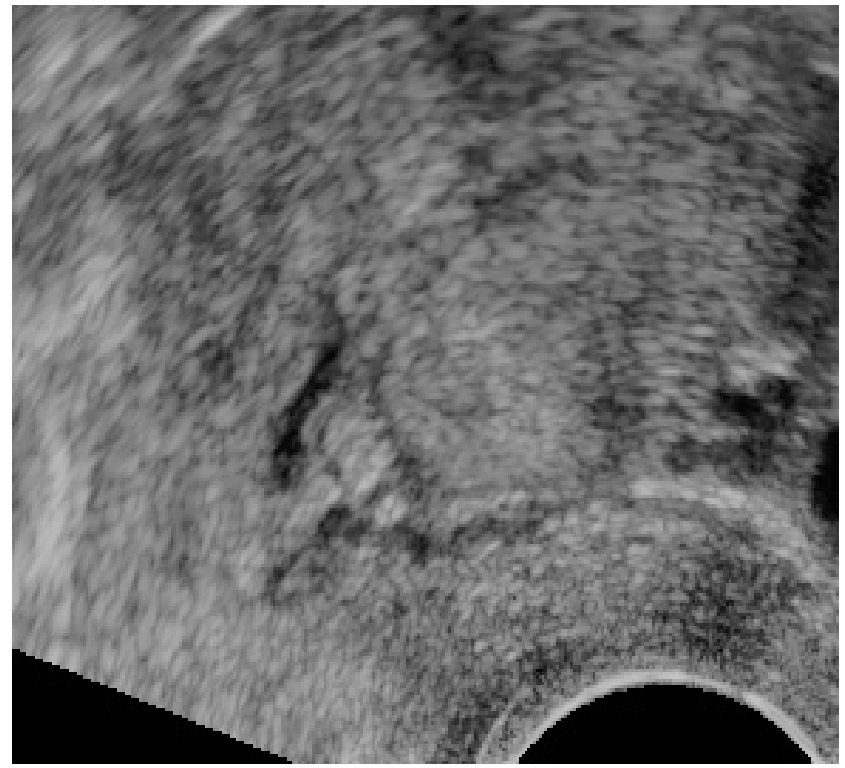

Figura 6. Gestación día 18 (Gestation day 18). 


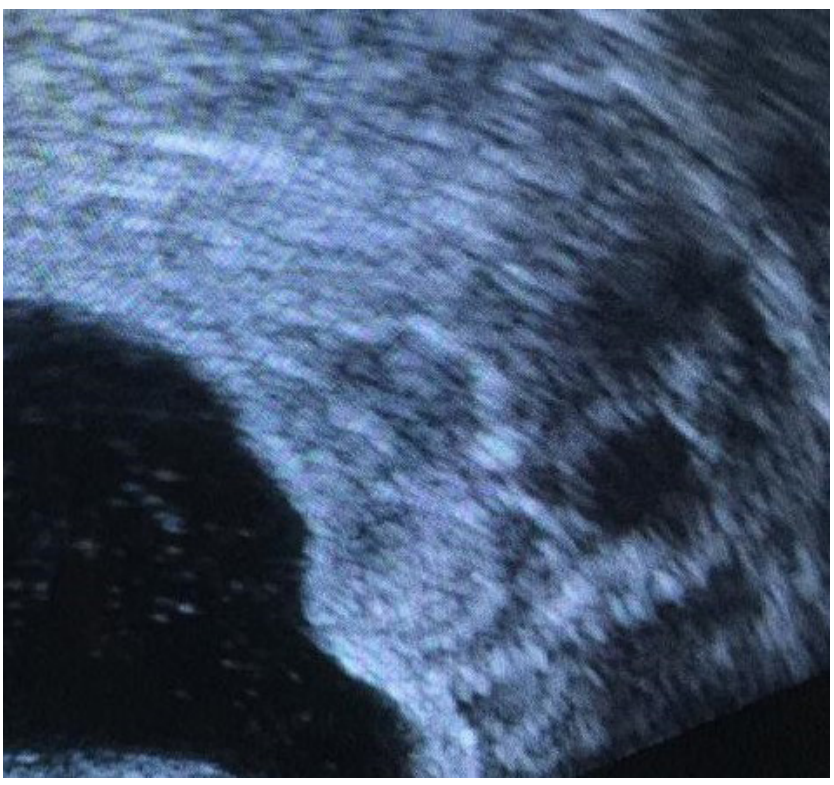

Figura 7. Gestación dia 19. Vesícula embrionaria ya identificable (Gestation day 19. Embryonic vesicle already identifiable.)

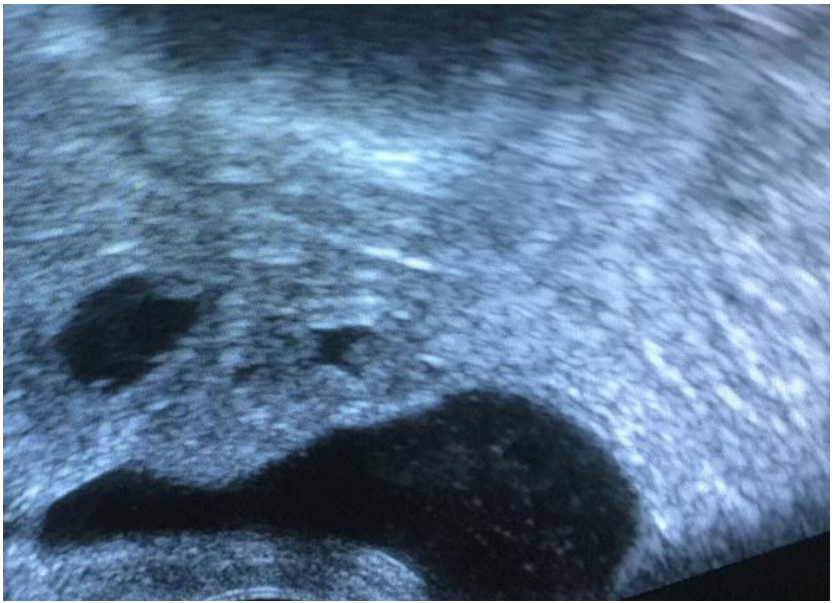

Figura 8. Gestación 21 días. Vesícula embrionaria ya identificable (Gestation 21 days. Embryonic vesicle already identifiable.)

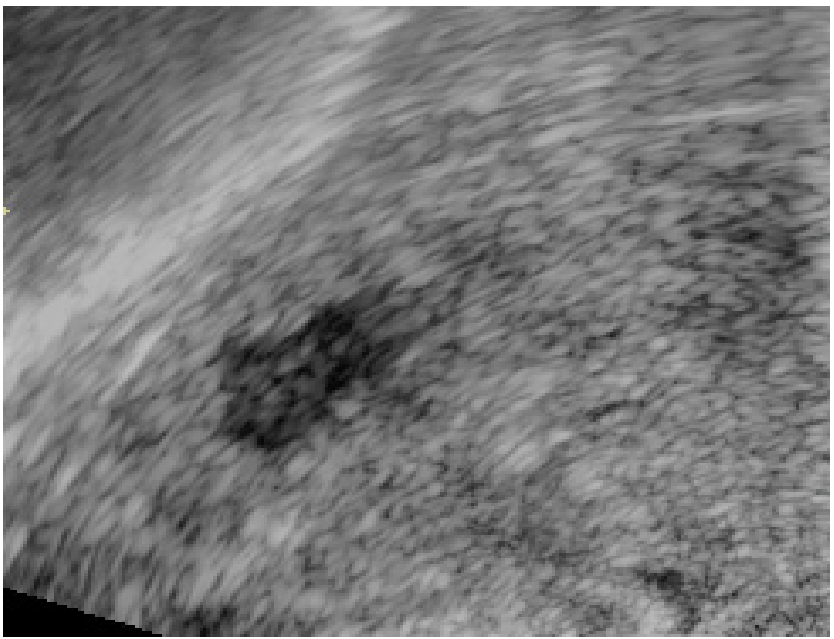

Figura 9. Gestación día 23. Vesícula embrionaria identificable (Gestation day 23. Identifiable embryonic vesicle.) tanto el latido cardiaco del embrión como el de cordón umbilical son perfectamente detectables, permitiendo confirmar la viabilidad embrionaria (Figuras 14 y 15). A partir del día 40 de gestación apreciamos que los exámenes se realizan mejor mediante la sonda transabdominal, ya que nos permite una visión más amplia de todo el campo a examinar, pudiendo visualizar también embriones que están en planos más difíciles de acceder con la sonda endovaginal, ya que el campo de visión de esta última es mucho más reducido.

El estudio está en fase preliminar, pero ya podemos comprobar que es posible un diagnóstico certero de gestación el día 25, con confirmación de viabilidad embrionaria, lo que permitirá la separación de hembras gestantes de vacías y el planteamiento reproductor de la ganadería. Con la evaluación de un mayor número de animales pretendemos establecer con seguridad el día más temprano de detección de gestación y comprobar si es posible un diagnóstico certero antes del día 18. También se pretende continuar la evaluación de la gestación hasta el parto, y comprobar la fecha de aparición de estructuras como placentomas o la medición de apéndices y órganos fetales. Una vez paridas, sería interesante descubrir lo que tardan las hembras caprinas de esta raza en volver a ciclar y el estado y evolución tanto del útero como de los ovarios.

\section{OTROS PARÁMETROS QUE NOS PERMITE ESTUDIAR LA ULTRASONOGRAFÍA.}

El uso de la ecografía no solo es útil en el diagnóstico de la gestación y viabilidad fetal, también puede utilizarse a lo largo de la misma para comprobar su evolución y proporcionar información que puede ser de utilidad para el manejo del rebaño como el conocimiento del sexo fetal de los cabritos que se esperan.

La determinación del sexo fetal se realiza mediante la localización del tubérculo genital (TG) y su posición con respecto al cordón umbilical y las extremidades inferiores. El TG se visualiza como una pequeña estructura hiperecogénica, con forma de grano de arroz, a partir del día 55 de gestación, cuando ya ha migrado a su posición definitiva (Amer, 2010; Santos et al. 2018).

El uso de la ecografía también resulta útil para determinar el tiempo de gestación y predecir por tanto la fecha de parto en los casos en los que no se sabe con exactitud la fecha en la que se produjo la fecundación. Los equipos avanzados de ultrasonografía nos permiten medir estructuras fetales, cuyo tamaño está correlacionado con la edad fetal. Así Lee et al. (2005) determinaron que el diámetro del tronco embrionario y la medida de los ejes cardiacos estaban estrechamente relacionados con la edad fetal en la cabra Coreana negra, mientras que otros autores correlacionan estrechamente el crecimiento de diámetro biparietal con la edad fetal (Haibel, 1988; Reichle y Haibel, 1991).

\section{ECOGRAFÍA 3D}

Si bien la ecografía 2-D nos proporciona imágenes de planos bidimensionales, con la tecnología 3-D podemos obtener una imagen en cualquier plano del espacio de un órgano o región de interés. Las imágenes bidimensionales obtenidas por el transductor ultrasónico se orientan en el espacio. El examinador desplaza 


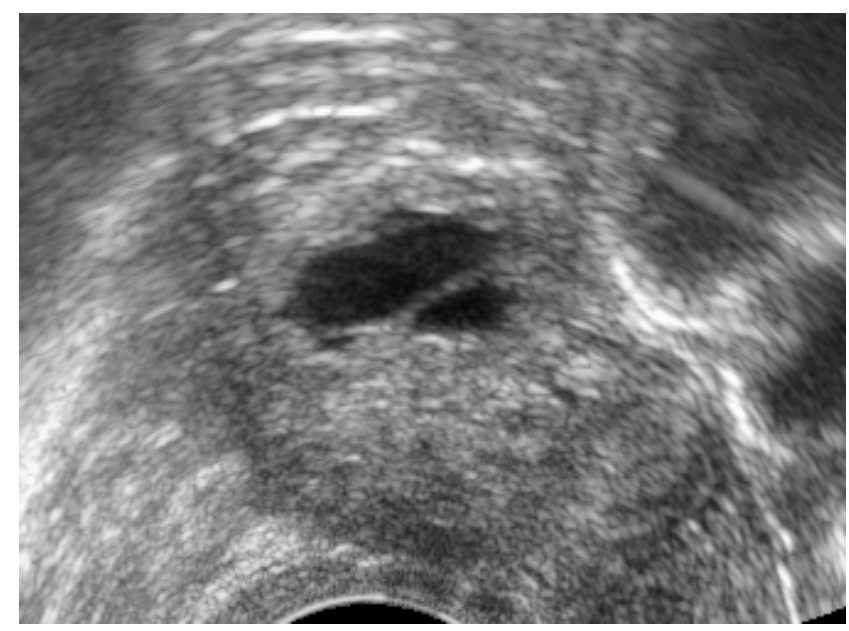

Figura 10. Gestación día 25. Vesícula embrionaria (Gestation day 25. Embryonic vesicle.)

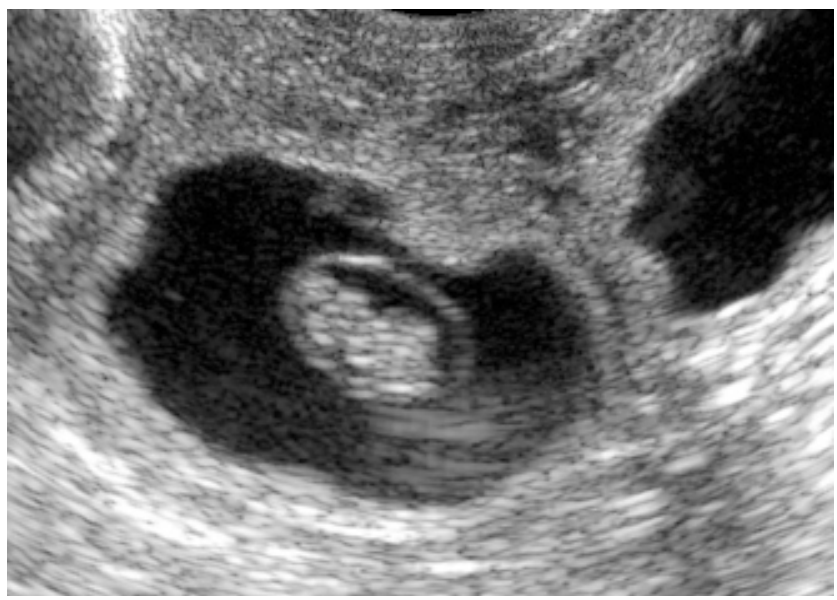

Figura 11. Embrión caprino en día 30, rodeado de membrana amniótica (Caprine embryo on day 30 , surrounded by amniotic membrane).

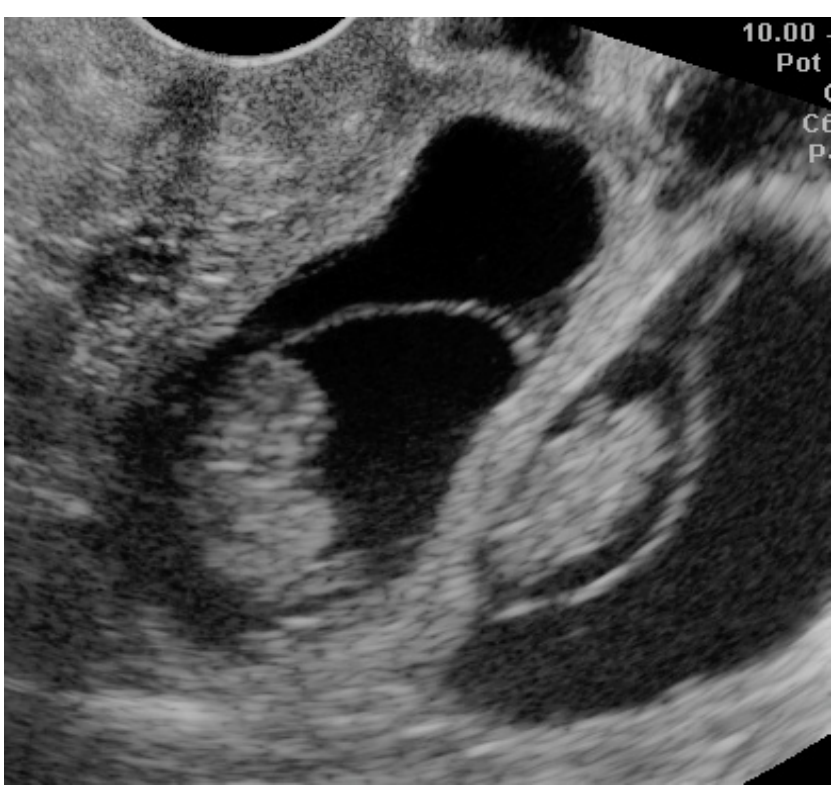

Figura 12. Gestación doble en día 35 (Double gestation on day 35.$)$

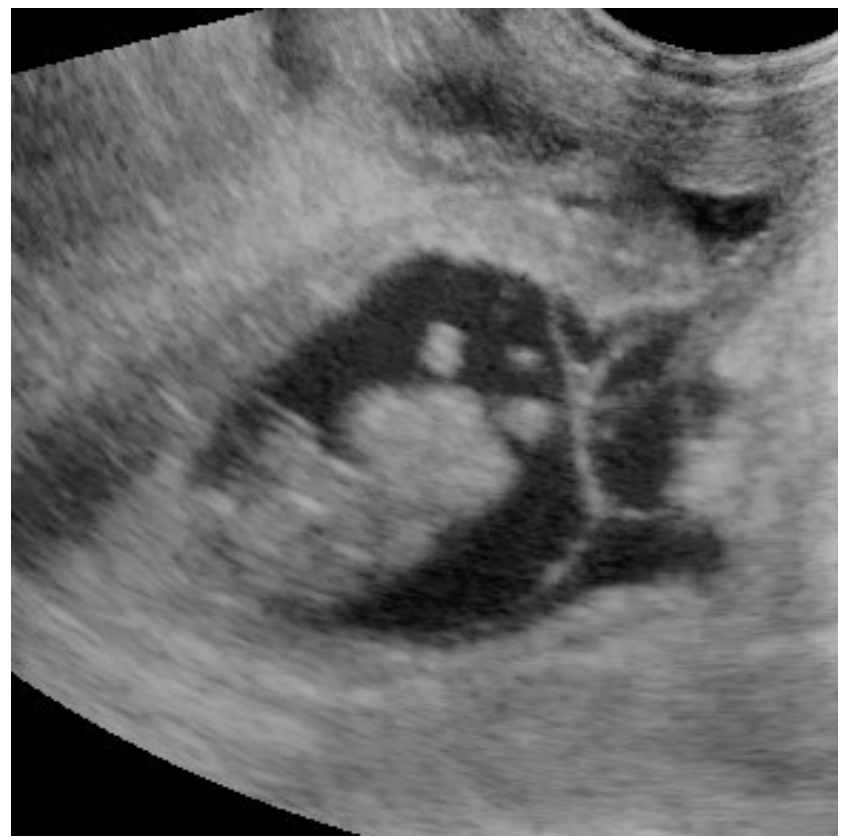

Figura 13. Embrión caprino en día 40, ya se aprecian las extremidades inferiores y la cola (flechas) (Caprine embryo on day 40, the lower limbs and tail (arrows) are already appreciated.)

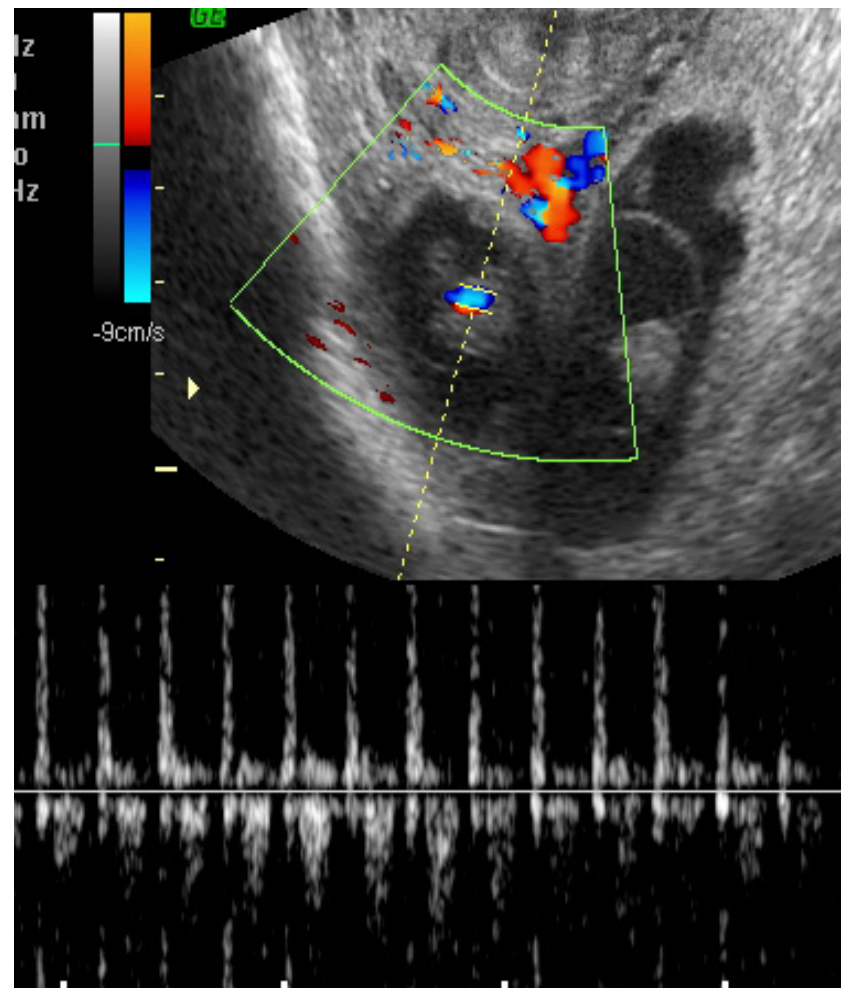

Figura 14. Latido cardiaco fetal en día 35, detectado mediante sistema Doppler (Fetal heartbeat on day 35, detected by Doppler system.)

la sonda mediante movimientos de traslación o rotación obteniendo una secuencia de imágenes 2-D que son almacenadas en un ordenador acoplado al ecógrafo donde son posteriormente procesados mediante un programa informático. Obtenemos un conjunto de imágenes que reconstruyen un volumen (Alcázar, 2005). 


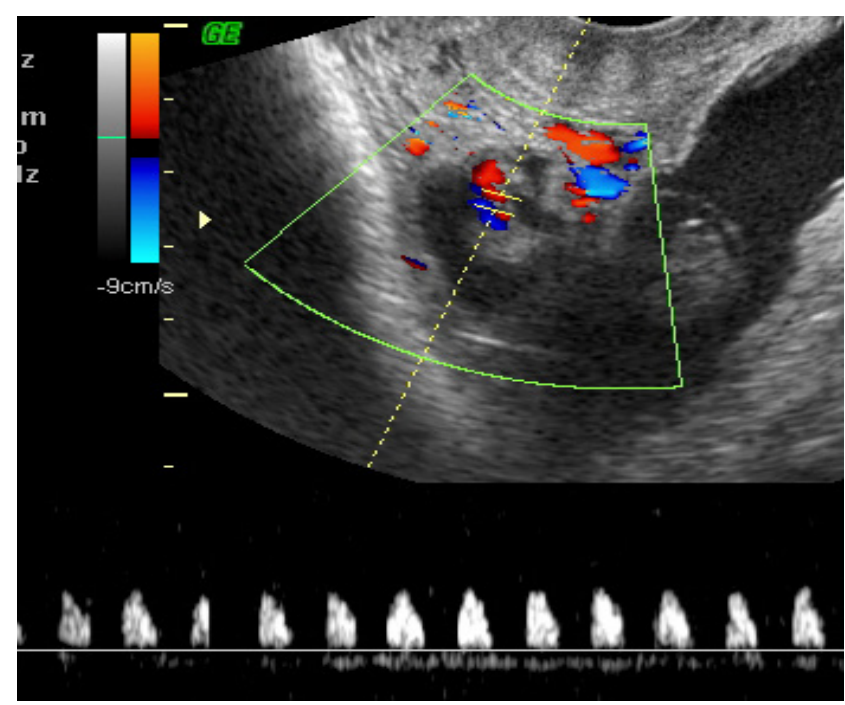

Figura 15. Flujo sanguíneo de cordón umbilical, detectado mediante sistema Doppler en día 35 (Umbilical cord blood flow, detected by Doppler system on day 35.)

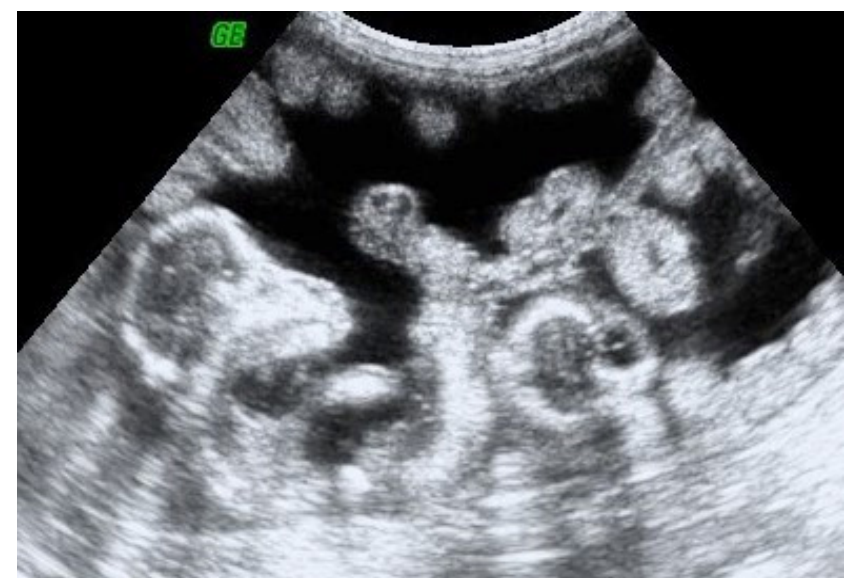

Figure 16. Feto caprino Murciano-Granadino de 67 días. Se aprecian los placentomas (flechas) y podemos distinguir en el interior del cráneo hiperecogénico el cerebro, con distinta ecogenicidad (Murciano-Granadino caprine fetus of 67 days. The placentomas (arrows) are appreciated and we can distinguish inside the hyperecogenic skull the brain, with different ecogenicity.)

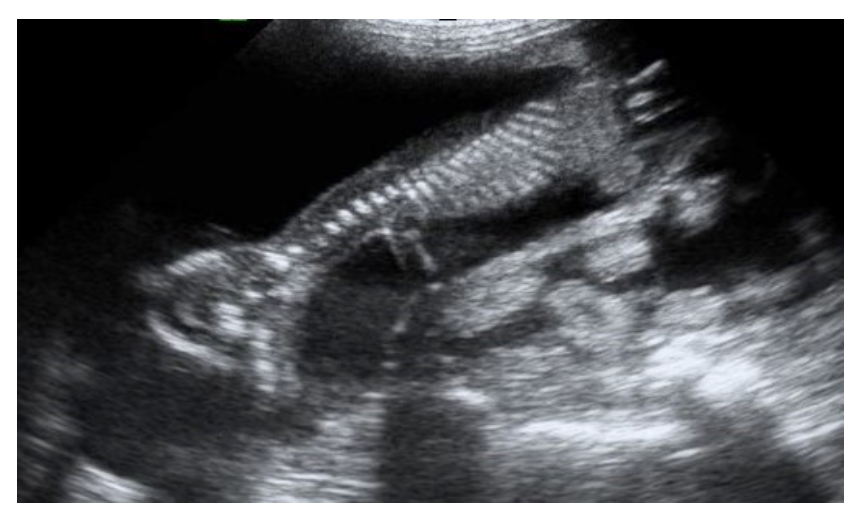

Figura 17. Feto caprino de 72 días. Se aprecia claramente toda la estructura ósea del cráneo, columna vertebral y costillas como estructuras muy ecogénicas (72-day caprine fetus. The entire bone structure of the skull, spine and ribs is clearly seen as highly ecogenic structures.)
Cuando las imágenes en 3-D se presentan en tiempo real, hablamos de ecografía en 4-D (Markov y Dimitrova, 2006). Su uso en veterinaria no está muy extendido, debido al alto coste de los equipos necesarios y a que el animal debe estar muy quieto durante el examen, para evitar artefactos en la imagen final, por lo que se recomienda la sedación o bien, una fuerte sujeción (Chandolia y George, 2011). La mayoría de autores coinciden en afirmar que es una metodología complementaria a la evaluación 2-D y no sustitutiva de esta, y que resulta especialmente útil en la detección de malformaciones fetales tempranas (Karadaev et al., 2019) y para visualizar con mayor detalle las distintas capas de la placenta y de la unión del feto al endometrio. También nos da una imagen más detallada de la superficie del embrión a partir del día 24 de gestación (Kumar et al., 2015). La ecografía 5-D, de uso reciente en reproducción humana, no es sino la 4-D bastante mejorada. Se trata de un software o programa, HD Live, que mejora la calidad de la imagen para que podamos tener una imagen mucho más real del feto. Consiste en la introducción de una serie de técnicas de filtrado que reducen las manchas, eliminan el ruido de las imágenes y realzan los bordes de los tejidos, destacando y priorizando las formas y estructuras del feto (babycine.com). Debido a que su utilidad actual es meramente emocional, y que son necesarios equipos modernos y costosos, no se utiliza en ecografía veterinaria.

\section{CONCLUSIONES}

El uso de la ecografía es un método muy útil en la detección temprana de gestación en caprinos, pudiendo diferenciar a hembras gestantes de vacías 25 días tras la monta/inseminación, además de permitir la valoración del sistema reproductor de las hembras o el número de fetos esperados. El conocimiento de todos estos parámetros hará más eficiente el manejo reproductivo del rebaño y ayudará a mejorar el rendimiento económico de la explotación.

Ecógrafos más avanzados permiten también la evaluación de la viabilidad fetal mediante detección de latido cardiaco incluso antes de poder ver movimientos fetales, o determinar el sexo fetal. El mayor inconveniente es que son equipos costosos que no suelen presentarse en granjas, pero son de utilidad con fines de investigación, cuyos resultados podrán ser aplicados en la práctica de cualquier explotación ganadera caprina.

El uso de la ecografía 3D/4D podría complementar a la de tipo 2-D para ayudar en diagnóstico y evolución de la gestación en caprinos. Son necesarios estudios con esta tecnología en la especie caprina, ya que no existen actualmente muchos estudios ecográficos en 3-D en cabras.

\section{AGRADECIMIENTOS}

Estos trabajos han sido financiados por Fondos Europeos de Desarrollo Regional (FEDER), mediante el proyecto de investigación Feder 1420-26, Titulado: “Los recursos genéticos animales resilientes al cambio climático. Mejora y diversificación de productos alimenticios derivados obtenidos de forma biosostenible". 


\section{BIBLIOGRAFÍA}

Alcázar, J.L. (2005). Fundamentos de la ecografía tridimensional (artículo de revisión). Rev. Med. Univ. Navarra/ vol 49, n 4. Pp: 9-12.

Amer, H.A. (2010). Ultrasonographic assessment of early pregnancy diagnosis, fetometry and sex determination in goats. Animal Reproduction Science, 117(3-4), 226-231.

Arashiro, E.K.N.; Ungerfeld, R.; Clariget, R.P.; Pinto, P.H.N.; Balaro, M.F.A.; Bragança, G.M. \& Brandão, F.Z. (2018). Early pregnancy diagnosis in ewes by subjective assessment of luteal vascularisation using colour Doppler ultrasonography. Theriogenology. 106, Pp: 247-252.

Basaran, D.A. (1999). Diagnosis of Ovulation Rate and Embriyonal, Foetal Development by Transrectal Ultrasonography in the White Goats. Turkish Journal of Veterinary and Animal Sciences, 23(6), 567-574.

Bo, G.A. y Caccia, M. (2000). Ultrasonografía reproductiva en el ganado bovino. Revista Taurus, 2. Pp:23-39.

Bidinost, F.; Gibbons, A.E.; Cueto, M. y Norte, M.P. (1999). Ecografía para el diagnóstico de preñez en ovinos y caprinos. INTAEEA Bariloche Macroregión Patagonia Norte. [En línea]. http://esa.ipb.pt/ ntecvet/Grupos_tarba/equipa_reproducao/Apontmentos_Varios/ ecografia_diagnostivo_gestacao_tecnologia.pdf

Brant, W.E. \& Dougherty, R.S. (2012). Vascular Ultrasound. Fundamentals of Diagnostic Radiology, 3, 954-87.

Chandolia, R.K. and George, J. (201 1). Application of 3D/4D ultrasonography in canine gynaecology and obstetrics. Indian Journal of Canine Practice, Vol. 3(2). Pp: 99-102

Cosentino, I.O., Balaro, M.F.A., Leal, F.S.C., da Silva Carvalho, A.B., de Souza, P.R.C., Arashiro, E.K.N., \& Brandão, F.Z. (2018). Accuracy of assessment of luteal morphology and luteal blood flow for prediction of early pregnancy in goats. Theriogenology, 121, 104-111.

Díez, N. (1992). Principios básicos de la ecografía (artículo de revisión).

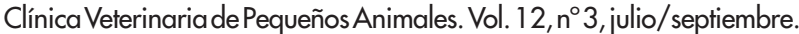

Erdogan, G. (2012). Ultrasonic assessment during pregnancy in goats-a review. Reproduction in domestic animals, 47(1), 157-163.

Fricke, P.M. (2002). "Scanning the future ultrasonography as a reproductive management tool for dairy cattle". J. dairy Sci. 85: 1918-1926.

Ginther, O.J. (2007). Ultrasonic Imaging and Animal Reproduction: ColorDopplerUltrasonography. Book 4 Equiservices Publishing, Wisconsin.

Giraldo, C. (2003). Principios básicos de ultrasonografía veterinaria. Revista MVZ Córdoba, 8(2). https://doi.org/10.21897/rmvz.508

Goel, A.K. \& Agrawal, K.P. (1992). A review of pregnancy diagnosis techniques in sheep and goats. Small Ruminant Research, 9, 255-264.

Gonzales, F.; Cabrera, F.; Batista, M.; Rodriguez, N.; Alamo, D.; Sulon, J.; Beckers, J.F.; Gracia, A. (2004). A comparison of diagnosis of pregnancy in the goat via transrectal ultrasound scanning, progesterone and pregnancy-associated glycoprotein assays. Theriogenology 62, $1108-1115$.

Griffin, P.G. \& Ginter, O.J. (1992). Research applications of ultrasonic imaging in reproductive biology. J. Anim. Sci. 70: 953-972.

Groenenberg, A.; Fernández, G.y Catalano, R. (2016). Uso de la ecografía en ovinos como método de dignóstico de gestación. Descripción de la metodología y análisis de resultados. Facultad de ciencias veterinarias UNCPBA. Tesina fin de grado. https://docplayer.es/57537763-Usode-la-ecografia-en-ovinos-como-metodo-de-diagnostico-de-gestaciondescripcion-de-la-metodologia-y-analisis-de-resultados.html

Haibel, G.K. (1988). Real-time ultrasonic fetal head measurement and gestational age in dairy goats. Theriogenology 30. Pp: 1053-1057.

Karadaev, M.; Fasulkov, I.; Vasilev, N.; Hristov, K. and Fedev, I. (2019). Three dimensional (3D) ultrasound investigations for monitoring of the second and third pregnancy trimester in goats. TMVM, 4(2). Pp: 72-76.

Karen, A.; Samir, H.; Ashmawy, T. \& El-Sayed, M. (2014). Accuracy of $B$-mode ultrasonography for diagnosing pregnancy and determination of fetal numbers in different breeds of goats. Animal reproduction science, 147(1-2), 25-31.

Kumar, K.; Chandolia, R.K.; Kumar, S.; Pal, M. and Kumar, S. (2015). Two-dimensional and three-dimensional ultrasonography for pregnancy diagnosis and antenatal fetal development in Beetal goats, Vet. World 8(7): Pp: 835-840.
Lavoir, M.C. and Taverne, M.A.M. (1989). The diagnosis of pregnancy and psuedopregnancy and the determination of foetal number in goats, by means of real-time ulterasound scanning. In: Diagnostic Ultrasound an Animal Reproduction, Taverne MM and AH Willemse (Eds.). Kluwer Academic Publisher, Dordrecht, Pp: 89-96.

Lee, Y.; Lee, O.; Cho, J.; Shin, H.; Choi, Y.; Shim, Y.; Choi, W.; Shin, H.; Lee, D.; Lee, G. and Shin, S. (2005). Ultrasonic Measurement of Fetal Parameters for Estimation of Gestational Age in Korean Black Goats. J. Vet. Med. Sci. 67(5). Pp: 497-502.

Markov, D. and Dimitrova, V. (2006). Prenatal diagnosis of structural anomalies of the fetus by 3D/4D ultrasound. Akusherstvo i Ginecologiia, Vol. 45(Suppl. 3): Pp: 32-36.

Martinez, M.F.; Bosch, P.; Bosch, R.A. (1998). Determination of early pregnancy and embryonic growth in goats by transrectal ultrasound scanning. Theriogenology 49, 1555-1565.

Medan, M.S. \& Abd El-Aty, A.M. (2010). Advances in ultrasonography and its applications in domestic ruminants and other farm animals reproduction. Journal of Advanced Research, 1(2), 123-128.

Memon, M.A. \& OH, R.S. (1980). Methods of pregnancy diagnosis in sheep and goats. Cornell Veterinarian, 70, 226-231

Moreno, A.; López, J.; Sánchez, M.A.; Chacón, F. y Hervás, J. (1999). Manual práctico de ecografía comparada en pequeños animales. Libro editado por Colegio de Veterinarios de Málaga.

Peinado, B. y Sánchez, M.C. (1998). Eficacia de la inseminación artificial caprina. Comparación con la monta natural, influencia del macho de raza Murciano Granadina (M-G). VII Congreso Nacional y IV Latinoamericano de Etología. Comunicación tipo póster. Organizado por la Sociedad Española de Etología. Pontevedra, 7-10 de septiembre de 1998.

Pellerito, J.; \& Polak, J.F. (2012). Introduction to Vascular Ultrasonography E-Book. Elsevier Health Sciences.

Petridis, I.G.; Barbagianni, M.S.; loannidi, K.S.; Samaras, E.; Fthenakis, G.C. \& Vloumidi, E.I. (2017). Doppler ultrasonographic examination in sheep. Small Ruminant Research, 152, 22-32.

Poto, A.; Peinado, B.; Lorenzo, M.; Domínguez, M.C.; Gergatz, E.; Göker, E.; Bali Pap, A. (1995). Inseminación artificial en caprino. Aportaciones en ganado de raza Murciano-Granadina. Mundo Ganadero/n ${ }^{\circ} 12 /$. Págs. 46-50.

Ramírez Videla, R.; Robson, C.; Aguilar, D.; Benítez, J. (2009). Ecografía: Herramienta para el ordenamiento productivo de la majada. Instituto Nacional de Tecnología Agropecuaria. $N^{\circ} 450$. ISSN N ${ }^{\circ}$ 0327-3059. http://inta.gob.ar/sites/default/files/script-tmp-inta_ecografa_en_ ovinos.pdf

Reichle, J.K. and Haibel, G.K. (1991). Ultrasonic biparietal diameter of second trimester pygmy goat fetuses. Theriogenology. 35. Pp: 689-694.

Sánchez, R. y Alfonso, E. (2000). Ultrasonografía en reproducción animal. TECNOVET: año 6, n⿳ 1, marzo.

Santiago-Moreno, J.; González-Bulnes, A.; García-López, M.; LópezSebastián, A.; (1995)a. Diagnóstico precoz de gestacióny determinación del número de embriones mediante ecografía transrectal en la cabra. ITEA 91A, 37-43.

Santiago-Moreno, J.; Gonzalez-Bulnes, A.; García-López, M. y LópezSebastián, A. (1995)b. Valoración de estadíos precoces de gestación en oveja y cabra mediante ecografía transrectal. Invest Agr 10, 53-61.

Santos, M.H.B.; Moura R.T.D.; Chaves, R.M.; Soares, A,T.; Neves, J.P.; Reichenbach, H.D.; Lima, P.F. and Oliveira, M.A.L. (2018). B-mode ultrasonography and ecobiometric parameters for assessment of embryonic and fetal development in sheep. Anim. Reprod. Sci. 197. Pp: 193-202.

Serin, G.; Gökdal, Ö.; Tarımcılar, T.; \& Atay, O. (2010). Umbilical artery doppler sonography in Saanen goat fetuses during singleton and multiple pregnancies. Theriogenology, 74(6), 1082-1087.

Taverne, M. \& Noakes, D. (2009). Pregnancy and its diagnosis. In: Veterinary Reproduction and Obstetrics, eds. D. E. Noakes, T. J. Parkinson \& G. C. W. England, Saunders, Philadelphia, pp. 76-122 http://www.babycine.com 